\title{
A unique finding of cavum velum interpositum colloid-like cyst and literature review of a commonplace lesion in an uncommon place
}

This article was published in the following Dove Press journal: International Journal of General Medicine

\author{
Abdulaziz Mohammad \\ Al-Sharydah' \\ Sari Saleh Al-Suhibani' \\ Abdulrahman Hamad \\ Al-Abdulwahhab' \\ Mohammad Saad Al-Aftan' \\ Ahmad Fouad Gashgari \\ 'Diagnostic and Interventional \\ Radiology Department, King Fahd \\ Hospital of the University, Imam \\ Abdulrahman Bin Faisal University, \\ Dammam, Eastern Province, Saudi \\ Arabia; ${ }^{2}$ Diagnostic Radiology \\ Department, Dammam Central \\ Hospital, Dammam, Eastern Province, \\ Saudi Arabia
}

\begin{abstract}
Colloid cysts typically reside within the area of the anterior third ventricle, in the proximity of the foramen of Monro. Although they are considered to commonly localize in various parts of the cerebrum, they are exceedingly rare outside the ventricular system and rarely occur within the velum interpositum. We have reported here a rare case of a velum interpositum colloid-like cyst in a 23-year-old man, who presented to our clinic with temporary binocular strabismus, which he had been experiencing for the previous year. In addition, we have briefly reviewed evidence regarding the generation, anatomy, and pathogenesis of colloid cysts, as well as the management options for such rare cases. The present report is only the third to describe a colloid-like cyst located within the velum interpositum, providing additional data that may aid in elucidating the pathogenesis of these neoplasms.
\end{abstract}

Keywords: colloid cyst, computed tomography, magnetic resonance imaging, velum interpositum, third ventricle

\section{Introduction}

Colloid cysts are benign tumors of an enigmatic embryological origin, accounting for $\sim 1 \%$ of all intracranial neoplasms. They are typically found in the anterior portion of the roof of the third ventricle and may cause ventricular obstructive hydrocephalus by encroaching upon the Monro foramina. Although colloid cysts are congenital, they typically present between the third and fifth decades of life. ${ }^{1}$ Clinical manifestations progress rapidly, with symptoms attributable to increased intracranial pressure. Less commonly, memory deficits may occur due to forniceal compression. Later detection in children is also a feature of colloid cysts, with symptoms only becoming evident once full-blown hydrocephalus has developed. The classic imaging appearance of colloid cysts is attributed to the thick proteinaceous fluid they carry. ${ }^{2}$

Although colloid cysts are considered common in various parts of the cerebrum, they are exceedingly rare outside the ventricular system and rarely occur within the velum interpositum. ${ }^{3}$ Only two previous reports of pediatric patients in both sexes have described colloid cysts occupying the upper and lower strata of the tela choroidea, through which the internal cerebral veins and medial posterior choroidal arteries course. ${ }^{4,5}$

Here, we have discussed the first reported case of an adult male patient with such colloid-like cyst. Furthermore, the present report not only emphasizes the rarity of the diagnosis but also highlights the controversy regarding the precise germline derivation of these lesions.
Correspondence: Abdulaziz Mohammad Al-Sharydah

Diagnostic and Interventional Radiology Department, King Fahd Hospital of the University, Imam Abdulrahman Bin Faisal University, Dammam, Eastern Province, PO Box: 31952 (4398), Saudi Arabia Email amsharydah@iau.edu.sa 


\section{Case report}

A 23-year-old man with no known medical or surgical history presented to our ophthalmology clinic with reports of temporary binocular strabismus, which he had been experiencing for the past year. His ophthalmological examination was unremarkable, and no focal neurological deficits were observed. Routine laboratory investigations yielded normal results. However, computed tomography and magnetic resonance imaging of the head revealed a well-defined, rounded lesion $(2.4 \times 1.7 \times 1.5 \mathrm{~cm})$ within the velum interpositum. Relative to the gray matter, the lesion exhibited low to intermediate signal intensity on T1-weighted (Figure 1) and T2-weighted images. Low to intermediate signal intensity was also observed on fluid-attenuation inversion recovery images, relative to the signal in the adjacent cerebral parenchyma (Figure 2). No enhancement was observed in this region following intravenous injection of gadolinium, and neither diffusion restriction nor blooming artifacts were elicited using inhomogeneity sequences (Figure 3). In addition, we observed no perilesional edema, and there were no signs of hydrocephalus. No diffusion restriction was elicited on diffusion-weighted imaging (DWI)/apparent diffusion coefficient (ADC) sequences. Owing to the lesion's cystic nature, homogeneity, enhancement pattern, lack of calcification, and diffusion restriction, we limited the differential diagnosis of this lesion to a colloid-like cyst. A teratoma was thought to be less likely due to the homogeneity of the lesion, while

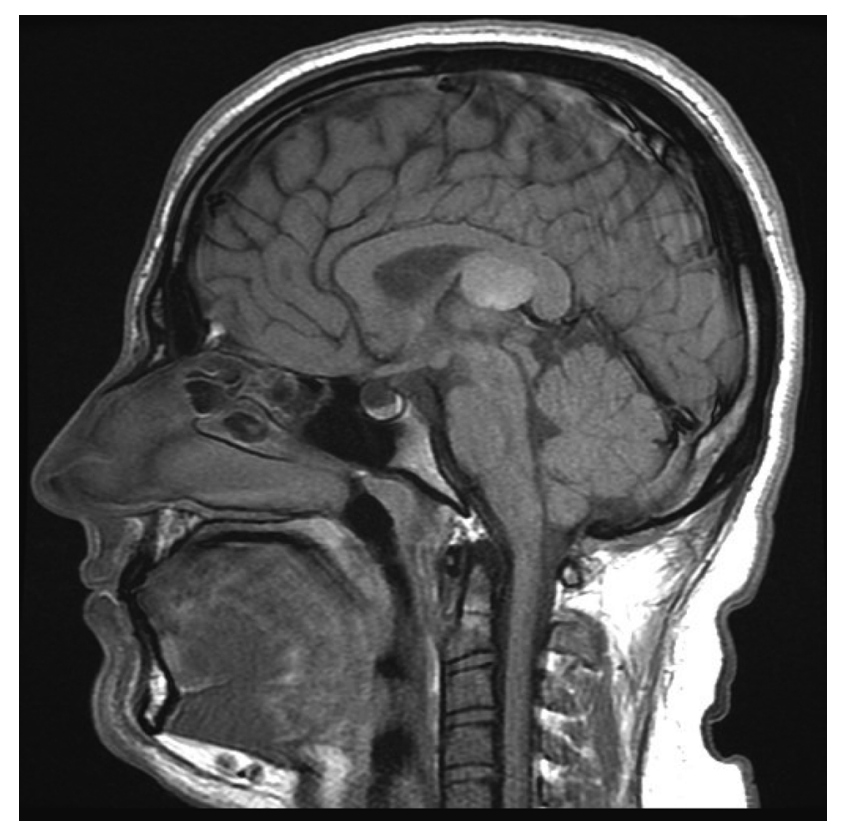

Figure I Mid-sagittal TI-weighted image of the brain shows a well-defined, rounded lesion at the roof of posterior third ventricle exhibiting low to intermediate signal intensity relative to the gray matter, with layering at the dependent portion. its enhancement characteristics excluded the likelihood of a pineal tumor. These characteristics are typical for colloid cysts. Moreover, these findings are entirely discordant with other possible diagnoses. Although excision of the characteristic colloid cyst using the transcallosal approach was recommended, the patient declined the procedure due to the stability of his condition and temporary nature of his symptoms. Following ophthalmological consultation, the use of prisms and vision therapy was recommended for palliative care. Unfortunately, the patient did not commit to the followup plan. He decided that the optical prisms were sufficient and did not complete the vision therapy program. He did not follow-up his appointments with the treating physician.

Consent for publication of the manuscript and the related patient information were obtained by King Fahd Hospital of the University, Imam Abdulrahman Bin Faisal University. Written informed consent was obtained from the patient for publication of the case details and accompanying images.

\section{Discussion}

Colloid cysts are benign, thin-walled, neuroepithelial intracranial neoplasms whose clinical, radiological, and pathological characteristics remain to be fully elucidated. Accounting for $\sim 1.5 \%$ of brain tumors, they usually reside between the columns of the fornices within the rostral portion of the third ventricle. The cysts are "attached" to a vascular pedicle originating from either the ependymal layer or choroid plexus, in proximity to the foramina of Monro. ${ }^{6,7}$ Colloid cysts have also been identified elsewhere within the brain, including the premedullary/pontine cistern, ${ }^{8}$ fourth ventricle, ${ }^{8}$ cerebellum, ${ }^{9}$ and within the region of the optic chiasma. ${ }^{10}$ Nonetheless, they can be found as single or paired cysts. ${ }^{11}$

A previous report by Desai et a ${ }^{12}$ described 105 cases of colloid cysts, although none were located within the region of the velum interpositum. To the best of our knowledge, only two cases of colloid cysts residing within the cistern of the velum interpositum have been reported. ${ }^{4,5}$ Moreover, the present case represents the first in which such lesions were identified in an adult male.

It remains unclear whether endodermal cysts containing colloidal material are pathogenetically or embryo-genetically similar to colloid cysts. ${ }^{11}$ While various theories regarding their origin have been proposed, most researchers believe that they are derived from endodermal neuroepithelial sources, such as the chordal epithelium and ependyma, but are not of a neuroglial origin. However, some authors have suggested that such colloid cysts derive from an endodermal source. ${ }^{13}$ A recent theory regarding the pathophysiology of familial 


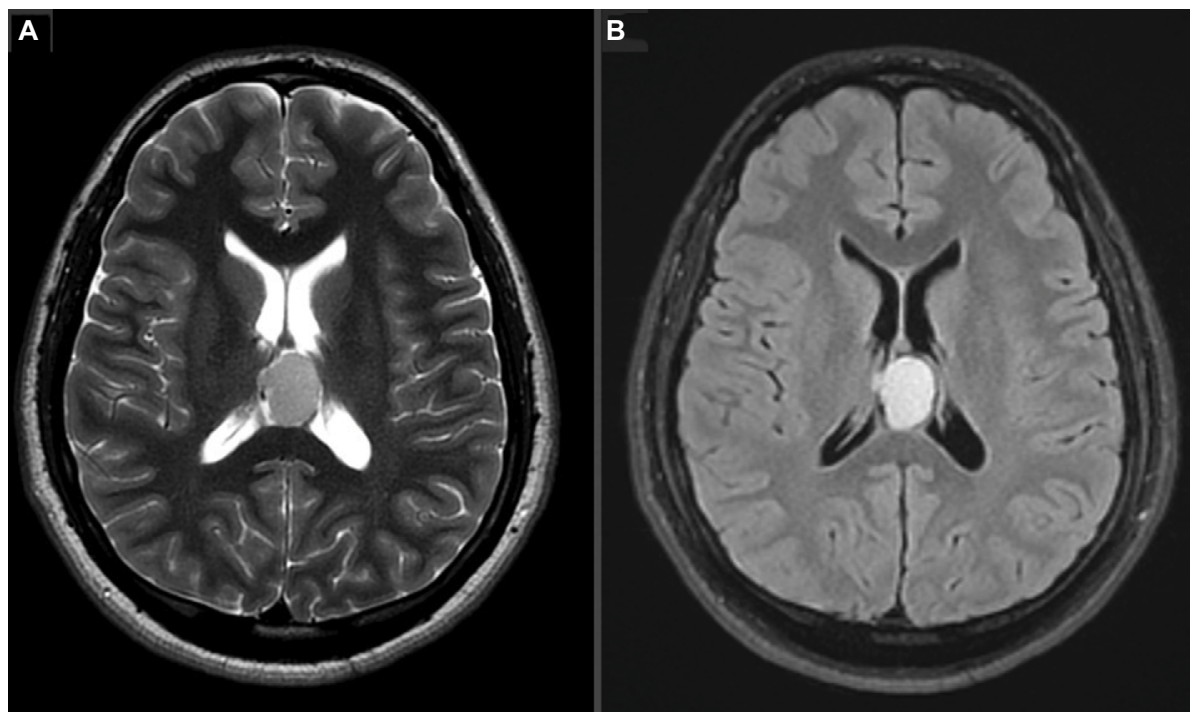

Figure 2 Axial T2-weighted (A) and fluid-attenuated inversion recovery (FLAIR) (B) images of the brain showing a well-defined, rounded lesion at the posterior part of the third ventricle.

Notes: The lesion exhibited homogenous, low to intermediate signal intensity and indented the wall of the lateral ventricle. The foramina of Monro were patent, with no signs of hydrocephalus.
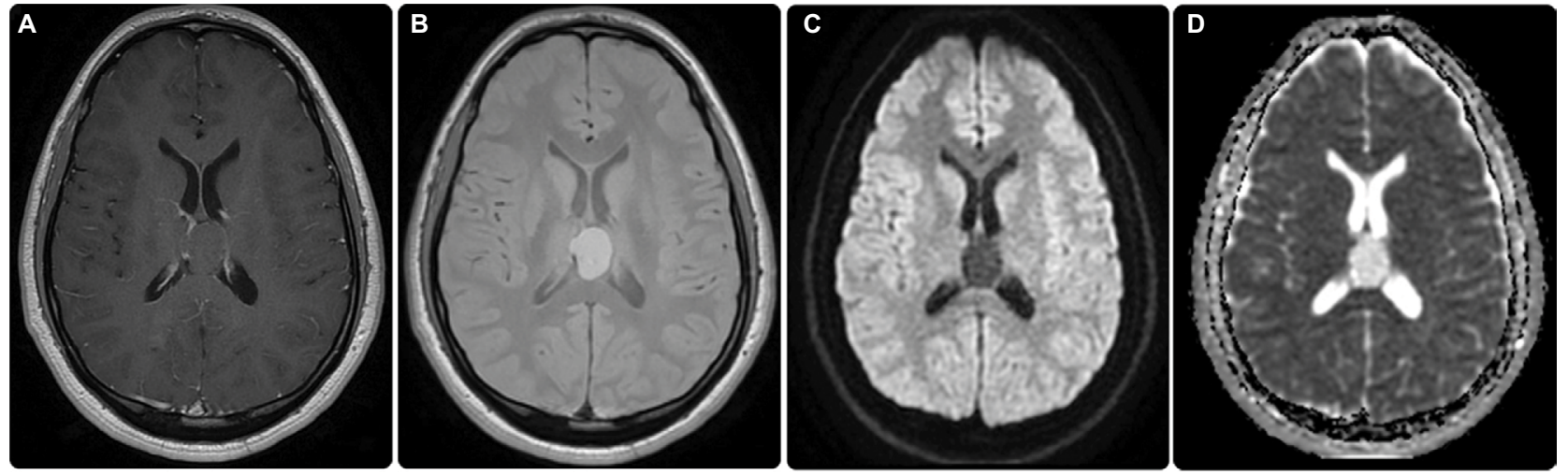

Figure 3 Post-contrast images showing no enhancement (A). Gradient images indicating no blooming artifacts to suggest hemorrhage or calcification (B). No diffusion restriction was elicited in diffusion-weighted imaging (DWI) (C)/apparent diffusion coefficient (ADC) (D) sequences.

colloid cysts of the third ventricle suggests an autosomal dominant inheritance. Moreover, evidence indicates that the pituitary-specific homeodomain factor may be involved in the development of colloid and Rathke's cleft cysts. ${ }^{14}$

Numerous cysts and cyst-like anatomic variants have been identified in the velum interpositum, ${ }^{15}$ including physiological enlargements and cysts of the cavum velum interpositum. Thus, differential diagnosis should also include meningioma, pineoblastoma/pinealocytoma, arachnoid cysts, and epidermoid cysts. ${ }^{16}$

Apart from a subtle peripheral (rim) enhancement depicted in our encountered lesion, no enhancement of the lesion was observed after gadolinium injection. Gra- dient images indicated no blooming artifacts to suggest hemorrhage or calcification. Moreover, magnetic resonance spectroscopy using single voxel sampling from the lesion revealed the absence of neural metabolites, thus denoting the extra-glial origin of the lesion. No diffusion restriction was elicited on DWI/ADC sequences. These characteristics are typical for colloid cysts.

Colloid cysts can be managed using various methods. Although a previous series of three cases indicated that some colloid cysts may spontaneously regress, ${ }^{17}$ excision via the posterior transcallosal approach allows for direct access to these neoplasms. In addition, approaching from the posterior parietal region avoids the motor cortex and enables access 
to the fornices, allowing for the excision of the cyst with a direct view of the internal cerebral veins.

The architecture, wall thickness, and imaging characteristics of the cystic lesion in the present case mimicked those of typical colloid cysts. Thus, we expect that the lesion's histological features would also match those reported in previous studies. The diagnosis of colloid cysts can be confirmed based on the features of flattened cuboidal epithelial cells/cytokeratins.

Hingwala et $\mathrm{al}^{5}$ recently described the first case of a colloid cyst in the velum interpositum in a 33-year-old woman who presented with headache and seizures. Clinically, our patient's condition manifested with temporary binocular strabismus. However, other manifestations may occur in patients with colloid cysts, such as crescendo headache, nausea, vomiting, cognitive or psychological disorders, and memory deficits.

The Colloid Cyst Risk Score (CCRS) is a recently developed grading system that aims to identify symptomatic lesions and stratify the risk of obstructive hydrocephalus during clinical decision-making. Surgical intervention is recommended for all patients with $\mathrm{CCRS} \geq 4$, as they exemplify the high-risk subgroup, with a sensitivity of $92 \%$ and a specificity of $93 \% .{ }^{18}$ Recent studies on the management of colloid cysts have compared endoscopic excision and microsurgical resection, demonstrating that the latter may be associated with a higher rate of complete resection, a lower rate of recurrence, and fewer repetitions. Nevertheless, morbidity is higher in the case of microsurgery than endoscopy. ${ }^{19}$ As such, other recent studies have advocated for combining endoscopic removal with stereotactic radiosurgery, which appears to be safe and effective, even in recurrent cases. Such studies have indicated that this strategy allows for $100 \%$ resection and is associated with favorable cognitive outcomes, a low risk of recurrence, and a low risk of morbidity. However, this method is disadvantageous in that it is longer and requires more complex instrumentation than conventional endoscopic resection. ${ }^{20}$

\section{Conclusion}

To our knowledge, the present report is the first to describe a colloid-like cyst located within the velum interpositum in an adult male patient. Although the exact germline derivation of colloid cysts remains controversial, an endodermal origin is considered most likely. In such rare cases, proper assessment and careful preoperative/surgical planning are required to prevent symptom recurrence.

\section{Author contributions}

All authors contributed toward data analysis, drafting and revising the paper and agree to be accountable for all aspects of the work.

\section{Disclosure}

The authors report no conflicts of interest in this work.

\section{References}

1. Gbejuade H, Plaha P, Porter D. Spontaneous regression of a third ventricle colloid cyst. Br J Neurosurg. 2011;25(5):655-657.

2. Kumar V, Behari S, Kumar Singh R, Jain M, Jaiswal AK, Jain VK. Pediatric colloid cysts of the third ventricle: management considerations. Acta Neurochir (Wien). 2010;152(3):451-461.

3. Rhoton AL Jr. The lateral and third ventricles. Neurosurgery. 2002;51(4 suppl):S207-S271.

4. Morris TC, Santoreneos S. Colloid cyst of velum interpositum: a rare finding: case report. J Neurosurg Pediatr. 2012;9(2):206-208.

5. Hingwala DR, Sanghvi DA, Shenoy AS, Dange NN, Goel AH. Colloid cyst of the velum interpositum: a common lesion at an uncommon site. Surg Neurol. 2009;72(2):182-184.

6. Beems T, Menovsky T, Lammens M. Hemorrhagic colloid cyst: case report and review of the literature. Surg Neurol. 2006;65(1):84-86.

7. Hadley DM. Colloid cyst of the IIIrd ventricle. J Neurol Neurosurg Psychiatry. 2002;72(2):15.

8. Goel A, Muzumdar D, Chagla A. Endodermal cyst anterior and anterolateral to the brainstem. A report of an experience with seven cases. $\mathrm{Br}$ J Neurosurg. 2005;19(2):163-166.

9. Müller A, Büttner A, Weis S. Rare occurrence of intracerebellar colloid cyst. Case report. J Neurosurg. 1999;91(1):128-131.

10. Killer HE, Flammer J, Wicki B, Laeng RH. Acute asymmetric upper nasal quandrantanopsia caused by a chiasmal colloid cyst in a patient with multiple sclerosis and bilateral retrobulbar neuritis. Am J Ophthalmol. 2001;132(2):286-288.

11. Thotakura AK, Nooti VSR, Ghanta S. Paired colloid cysts in the third ventricle. World Neurosurg. 2016;93:485.e7-e9.

12. Desai KI, Nadkarni TD, Muzumdar DP, Goel AH. Surgical management of colloid cyst of the 3rd ventricle-a study of 105 cases. Surg Neurol. 2002;57(5):295-302.

13. Ho KL, Garcia JH. Colloid cysts of the third ventricle: ultrastructural features are compatible with endodermal derivation. Acta Neuropathol. 1992;83(6):605-612.

14. Partington MW, Bookalil AJ. Familial colloid cysts of the third ventricle. Clin Genet. 2004;66(5):473-475.

15. Chen CY, Chen FH, Lee CC, Lee KW, Hsiao HS. Sonographic characteristics of the cavum velum interpositum. AJNR Am J Neuroradiol. 1998;19(9):1631-1635.

16. Vázquez-López ME, Pego-Reigosa R, Martínez-Vázquez F, ArmestoPérez V. Cysts in the velum interpositum. Rev Neurol. 2003;37(9):897.

17. Peeters SM, Daou B, Jabbour P, Ladoux A, Abi Lahoud G. Spontaneous regression of a third ventricle colloid cyst. World Neurosurg. 2016;90:704.e19-e22.

18. Beaumont TL, Limbrick DD Jr, Rich KM, Wippold FJ, Dacey RG Jr. Natural history of colloid cysts of the third ventricle. J Neurosurg. 2016;125(6):1420-1430.

19. Sheikh AB, Mendelson ZS, Liu JK. Endoscopic versus microsurgical resection of colloid cysts: a systematic review and meta-analysis of 1278 patients. World Neurosurg. 2014;82(6):1187-1197.

20. Birski M, Birska J, Paczkowski D, et al. Combination of neuroendoscopic and stereotactic procedures for total resection of colloid cysts with favorable neurological and cognitive outcomes. World Neurosurg. 2016;85:205-214. 
The International Journal of General Medicine is an international, peer-reviewed open-access journal that focuses on general and internal medicine, pathogenesis, epidemiology, diagnosis, monitoring and treatment protocols. The journal is characterized by the rapid reporting of reviews, original research and clinical studies across all disease areas.
The manuscript management system is completely online and includes a very quick and fair peer-review system, which is all easy to use. Visit http://www.dovepress.com/testimonials.php to read real quotes from published authors. 